\title{
Measurement of coupled spatiotemporal coherence of parametric down-conversion under negative group velocity dispersion
}

\author{
Paula Cutipa ${ }^{1,2, *}$, Kirill Yu. Spasibko ${ }^{1,2}$, and Maria V. Chekhova ${ }^{1,2,3}$ \\ ${ }^{1}$ Max Planck Institute for the Science of Light, Staudtstraße. 2, Erlangen D-91058, Germany \\ ${ }^{2}$ University of Erlangen-Nuremberg, Staudtstraße. 7/B2, 91058 Erlangen, Germany \\ ${ }^{3}$ Physics Department, Moscow State University, Leninskiye Gory 1-2, Moscow 119991, Russia \\ ${ }^{*}$ Corresponding author: paula.cutipa@mpl.mpg.de
}

\begin{abstract}
We present a direct measurement of the spatiotemporal coherence of parametric down-conversion in the range of negative group-velocity dispersion. In this case, the frequency-angular spectra are ring-shaped and temporal coherence is coupled to spatial coherence. Correspondingly, the lack of coherence due to spatial displacement can be compensated with the introduction of time delay. We show a simple technique, based on a modified Mach-Zehnder interferometer, which allowed us to measure time coherence and near-field space coherence simultaneously, with complete control of both variables. This technique will be also suitable for the measurement of second-order coherence, where the main applications are related to the two-photon spectroscopy.
\end{abstract}

Coherence is an important characteristic of light sources, including the ones producing non-classical light $1-3$. First-order coherence of a source of light is usually measured in an interferometer, by splitting the field in two (or more) parts and then overlapping the fields again, after a spatial displacement or temporal delay introduced between them.

The quantitative description of coherence is given by the first-order correlation function (CF) [4,5],

$$
G^{(1)}\left(t_{1}, t_{2}, r_{1}, r_{2}\right)=\left\langle E^{*}\left(t_{1}, r_{1}\right) E\left(t_{2}, r_{2}\right)\right\rangle,
$$

where $E\left(t_{i}, r_{i}\right)$ is the electric field at point $r_{i}$ and time $t_{i}, i=1,2$.

When the field is spatially uniform and stationary, the first-order CF depends only on the time delay and space displacement but not on the absolute values of time and space: $G^{(1)}\left(t_{1}, t_{2}, r_{1}, r_{2}\right)=G^{(1)}(\tau, \xi)$, where $\tau=t_{1}-t_{2}$ and $\xi=r_{1}-r_{2}$ are the time delay and space shift between the two fields. The standard way to measure the first-order $\mathrm{CF}$ in space and time is by using, respectively, the Young and Michelson interferometers [2].

Temporal and spatial first-order correlation functions are related, respectively, to the frequency spectrum $S(\omega)$ and the transverse wavevector spectrum $S(k)$ through Fourier transforms. These relations are known as the Wiener-Khinchine and van CittertZernike theorems 2]. Usually, the frequency - transverse wavevector spectrum is factorable, $S(\omega, k)=$ $S_{1}(\omega) S_{2}(k)$, which means that there is no coupling between temporal and spatial coherence and they can be studied separately. However, uncoupled spatiotemporal coherence is not always the case. Indeed, it has been demonstrated that the radiation produced through parametric down-conversion (PDC) has a non-factorable frequency-wavevector spectrum $S(\omega, k) \neq S_{1}(\omega) S_{2}(k)[6,7$. This leads to coupled spatiotemporal coherence described by the general- 
ization of the Wiener-Khinchin theorem, relating the first-order space-time correlation function with the frequency - transverse wavevector spectrum [8, 9]:

$$
G^{(1)}(\tau, \xi)=\iint S(\omega, k) e^{i k \xi-i \omega \tau} d \tau d \xi
$$

This property was reported experimentally for PDC in the range of positive group-velocity dispersion (GVD) 6], where the coupling between temporal and spatial coherence appears due to the 'X-shaped' frequency - transverse wavevector spectrum. The interesting consequence of the coupling between spatial and temporal coherence is that the reduction of coherence due to the spatial displacement can be compensated by the temporal delay and vice versa, which has been indeed demonstrated in Ref. [9]. However, the exact shape of $G^{(1)}(\tau, \xi)$ was not measured. Meanwhile, in the range of negative GVD $\left(\frac{d^{2} k}{d \omega^{2}}<0\right)$, the frequency-wavevector spectrum is very different. It becomes ring-shaped in the noncollinear nondegenerate case and spot-shaped in the collinear degenerate case 10,11 . Accordingly, coupled spatiotemporal coherence should be observed in this case as well, but the CF should be different. In particular, for a ring-shaped spectrum there is no collinear or degenerate emission. This feature should lead to a narrower first-order $\mathrm{CF}$ than in the X-shape case. In turn, the second-order CF should be also narrower in this case, which suggests very high spatial and temporal resolution in two-photon spectroscopy $11-14$.

In this work we directly measure the spatiotemporal first-order $\mathrm{CF}^{(1)}(\tau, \xi)$ for PDC in the negative GVD range. To this end, we develop a practical technique for measuring time and near-field space variables simultaneously. The measurement reveals coupled spatiotemporal coherence with typical coherence time on the order of femtoseconds and coherence radius on the order of micrometers. The same technique will be valid for the measurement of the second-order $\mathrm{CF}$, with applications related to two-photon absorption spectroscopy 14 16.

The frequency-wavevector spectrum of both highgain and low-gain PDC can be calculated from the longitudinal phase mismatch $\Delta k(\omega, k)$ using the

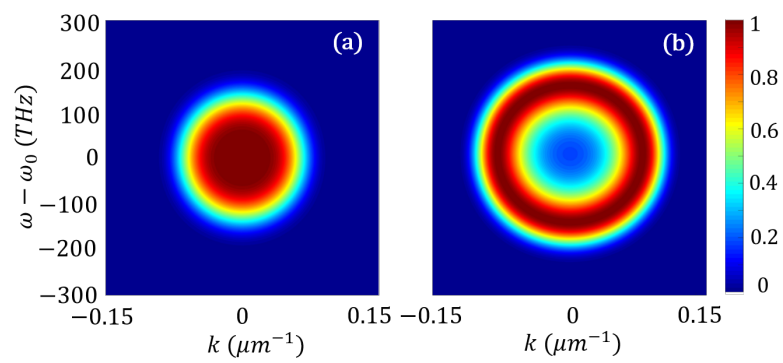

Figure 1: Calculated spectra $S(\omega, k)$ for PDC in a type-I BBO crystal pumped at $800 \mathrm{~nm}$ in (a) collinear degenerate (spot-shaped) and (b) noncollinear nondegenerate (ring-shaped) cases.

equation $7,17,18$

$$
S(\omega, k)=\left(\frac{G}{\mathcal{G}(\omega, k)} \sinh \mathcal{G}(\omega, k)\right)^{2},
$$

where

$$
\mathcal{G}(\omega, k)=\sqrt{G^{2}-\frac{(\Delta k(\omega, k) L)^{2}}{4}}
$$

is the parametric gain and $G$ is proportional to the effective second-order susceptibility and the pump field amplitude. Equation 3 was used to calculate the PDC spectra in the range of negative GVD (Fig. 1). For the calculation we assumed that PDC is pumped at $800 \mathrm{~nm}$ and the crystal is a $10 \mathrm{~mm} \beta$-bariumborate $(\mathrm{BBO})$ cut for type-I phase matching. In the collinear frequency-degenerate case, the spectrum $S(\omega, k)$ looks like a round spot as shown in Fig. 1 (a). It is broadband because the degenerate wavelength $1600 \mathrm{~nm}$ is close to zero-dispersion wavelength 10.

When the configuration changes to the noncollinear-nondegenerate one, the spectrum becomes nonfactorable and has a typical ring-like shape (Fig. 1 (b)). The size of the ring depends on the crystal orientation, i.e., the angle between its optic axis and the pump, and can be made very large. According to Eq. 2, in this case one can expect a non-factorable first-order $\mathrm{CF}$ with coherence time on the order of a few femtoseconds and coherence radius on the order of a few micrometers. 
The experimental setup for the CF measurement is shown in Fig. 2. It is based on a modified MachZehnder interferometer, enabling both a time delay and a space displacement between the two arms. To pump PDC, we used an amplified Ti-sapphire laser at $800 \mathrm{~nm}$ with a $1 \mathrm{~W}$ mean power, 1.6 ps pulse duration and $5 \mathrm{kHz}$ repetition rate. The beam size was reduced by a two-lens telescope, $L_{1}$ and $L_{2}$, to a waist of $600 \mu \mathrm{m}$. Type-I PDC was generated in an $L=10 \mathrm{~mm}$ BBO crystal. The parametric gain at zero phase mismatch was $G=6$. For the pump incident at $19.87^{\circ}$ to the optic axis, exact phase matching was satisfied for collinear frequencydegenerate PDC. The optic axis was in the $\mathrm{y}-\mathrm{z}$ plane; the pump beam was polarized along the y-direction (extraordinarily), as shown by a dot, while the downconverted radiation, along the x-direction (ordinarily), as shown by an arrow. For avoiding the spatial walk-off effects in the spectrum [19], all measurements were made with a $1.6 \mathrm{~mm}$ slit along the $\mathrm{x}$-direction placed in the Fourier plane of the lens $L_{3}$ with focal length $20 \mathrm{~cm}$. This reduced the angular spectra to one-dimensional, in the plane free from the walk-off. The pump was rejected using a dichroic mirror DM, and the down-converted beam was sent to the interferometer.

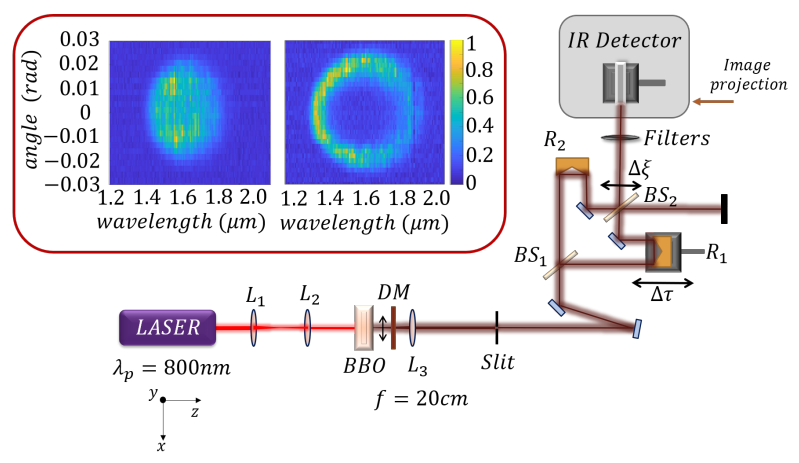

Figure 2: Experimental setup for measuring the firstorder correlation function. Time delay is introduced with the retroreflector $R_{1}$ and the space shift, with the beam splitter $B S_{2}$.

The inset of Fig. 2 shows the experimental wavelength-angular spectra $S\left(\lambda, \theta_{\text {ext }}\right)$ for the collinear degenerate (a) and noncollinear nondegenerate (b) cases. The angles $\theta_{\text {ext }}$ are external, i.e., measured outside of the crystal, and are related to the transverse wavevector $k$ as $\theta_{\text {ext }}=$ $k \lambda /(2 \pi)$. These spectra correspond to the theoretical frequency-wavevector distributions $S(\omega, k)$ shown in Fig. 1. The spectra were measured the same way as in Ref. [11]: by scanning in the far field the tip of a multimode fiber connected to a spectrometer, and recording the spectrum for each position of the fiber. The spectra correspond to the crystal orientation of $19.87^{\circ}$ (left) and $19.94^{\circ}$ (right).

The PDC beam was fed into the interferometer, consisting of two thin 50 : 50 beam splitters $B S_{1}$, $B S_{2}$ and two retroreflectors $R_{1}, R_{2}$. The time delay was varied by moving retroreflector $R_{1}$ and the space displacement, by shifting the second beam splitter $B S_{2}$. After the interferometer, the PDC beam passed through additional filters to remove the residual pump radiation, and was registered by a home-made infrared detector. The detector was based on an InGaAs PIN photodiode with a photosensitive area of $0.3 \mathrm{~mm}$ and a cutoff wavelength of $2.1 \mu \mathrm{m}$, which allowed us to measure the correlation properties without cutting the spectrum. For measuring the $\mathrm{CF}$ in the near field, we projected the output face of the crystal on the detector with lens $L_{3}$, placed at a distance of $43.5 \pm 0.1 \mathrm{~cm}$ from the BBO crystal. The resulting image had a magnification of 6.6.

The absolute value of the normalized first-order CF $g^{(1)}(\tau, \xi) \equiv G^{(1)}(\tau, \xi) / I$, with $I$ standing for the mean intensity, was measured as the interference visibility 20]. The latter was found by measuring the intensity at the output of the interferometer with the detector, whose sensitive area was small enough not to average over the interference fringes.

First, we measured the output intensity versus the time delay in the interferometer, with no space shift ( $B S_{2}$ fixed at the position of perfect alignment). The result is plotted in Fig. 3. The trace shows pronounced interference fringes, their period corresponding exactly to the degenerate wavelength 1600 $\mathrm{nm}$. The absolute value of normalized first-order CF $\left|g^{(1)}(\tau)\right|$ was found as the visibility of this interference pattern. 


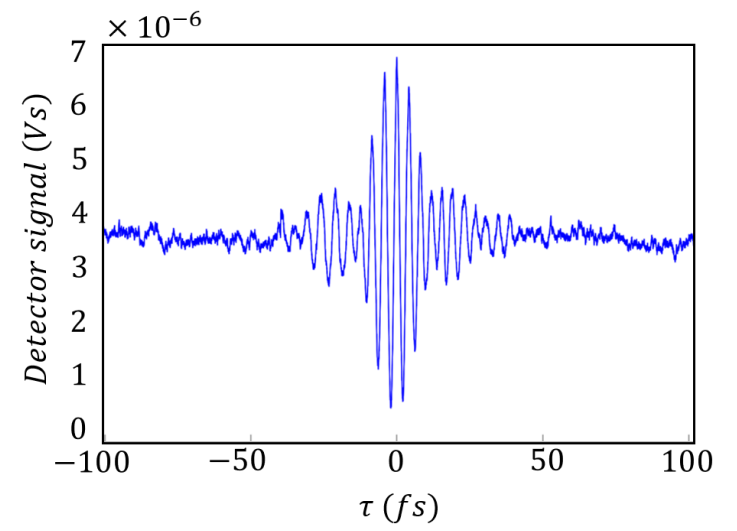

Figure 3: The detector signal measured versus the time delay in the interferometer. The crystal orientation is $19.94^{\circ}$. The fringe period corresponds to the wavelength $1600 \mathrm{~nm}$.

To perform the measurement of $\left|g^{(1)}(\tau, \xi)\right|$, the temporal delay and spatial displacement in the interferometer should be varied independently. Although the displacement of beamsplitter $B S_{2}$ introduces at the same time a temporal delay, this could be taken into account. To this end, we moved $B S_{2}$ in steps of $40 \mu \mathrm{m}$, and for each position the retroreflector $R_{1}$ was scanned with an automated translation stage. Each time, a profile as shown in Fig. 3 was measured, and shifted with respect to $\tau$ depending on the $B S_{2}$ position. Consequently applying this method, we were able to build the 2-D plot of $\left|g^{(1)}(\tau, \xi)\right|$ in time and space, as shown in Fig. 4. The measurements of $\left|g^{(1)}(\tau, \xi)\right|$ were performed for three crystal orientations, corresponding to the collinear degenerate and two noncollinear nondegenerate cases of PDC.

In Fig. 4 the left panels show the calculated plots of $\left|g^{(1)}(\tau, \xi)\right|$, whereas the right panels present the corresponding measured distributions. For the collinear degenerate case we use the crystal orientation $19.87^{\circ}(\mathrm{a}, \mathrm{b})$ and for the noncollinear nondegenerate case, $19.90^{\circ}(\mathrm{c}, \mathrm{d})$ and $19.94^{\circ}(\mathrm{e}, \mathrm{f})$. The peak at the center of all distributions indicates the trivial case of $\tau=0$ and $\xi=0$, where high coherence is evidenced. Experimentally, the visibility achieved is higher than the $80 \%$ for all the cases, which is good

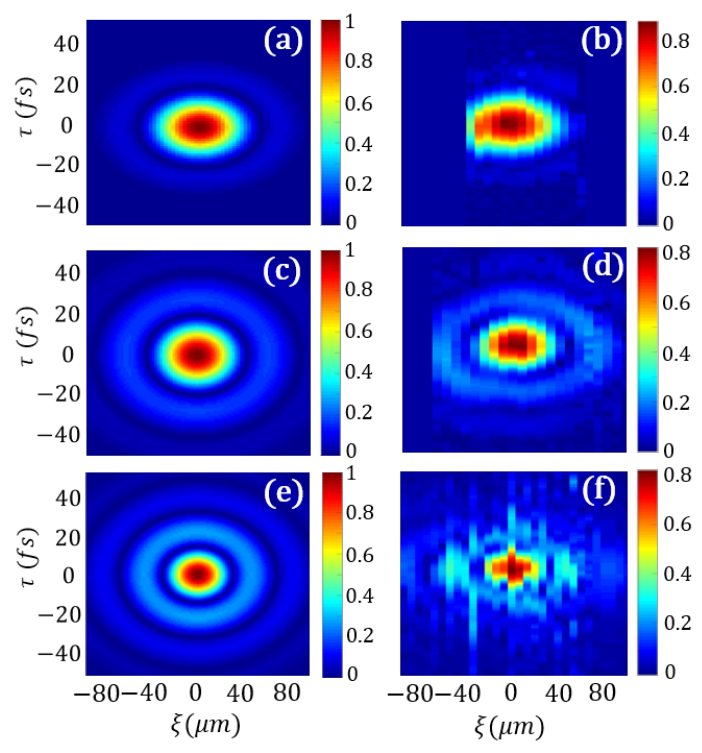

Figure 4: Calculated (a,c,e) and measured (b,d,f) distributions of $\left|g^{(1)}(\tau, \xi)\right|$ for the collinear degenerate $(\mathrm{a}, \mathrm{b})$ and noncollinear nondegenerate $(\mathrm{c}, \mathrm{d}, \mathrm{e}, \mathrm{f})$ PDC. The crystal orientations are $19.87^{\circ}(\mathrm{a}, \mathrm{b})$, $19.90^{\circ}(\mathrm{c}, \mathrm{d})$ and $19.94^{\circ}(\mathrm{e}, \mathrm{f})$.

enough for the observation of the coupled coherence exhibited in the weaker rings surrounding the central peak. For these rings, the lack of spatial coherence can be obviously compensated by the temporal delay and vice versa.

Figure 5 shows one-dimensional profiles of $\left|g^{(1)}(\tau, \xi)\right|$ from Fig. 4 (f): (a) as a function of $\xi$ with fixed $\tau=0$ and (b) as a function of $\tau$ with fixed $\xi=0$. The full widths at half maximum (FWHM) in these plots correspond to the coherence radius $\xi_{c}$ and coherence time $\tau_{c}$, respectively. The obtained values are $\xi_{c}=38 \pm 7 \mu \mathrm{m}$ and $\tau_{c}=16 \pm 2 \mathrm{fs}$, in good agreement with the theoretical numbers (see Table 1. where the experimental and theoretical coherence parameters are listed for all three studied orientations of the crystal).

From Table 1 we see that the coherence time and radius strongly depend on the crystal orientation. In particular, in the most noncollinear nondegenerate regime (large ring-shaped spectrum) the short- 

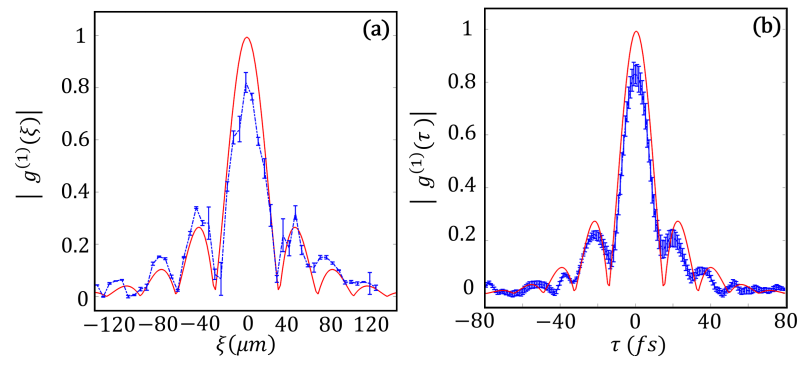

Figure 5: Experimental (blue) and calculated (red) one-dimensional 'cuts' of $\left|g^{(1)}(\tau, \xi)\right|$ from Fig. 4 (f): (a) $\left|g^{(1)}(\xi)\right|$ at $\tau=0$ and (b) $\left|g^{(1)}(\tau)\right|$ at $\xi=0$.

est coherence time and radius are achieved. This behaviour follows from the Fourier relation between $S(k, \omega)$ and $G^{(1)}(\xi, \tau)$ : the larger the ring-shaped spectrum, the shorter the coherence time and radius. In the case of the shortest coherence time and radius, as in panel (f) of Fig. 4 the finite resolution of the measurement of $1 \mathrm{fs}$ and $6 \mu \mathrm{m}$ reduced the quality of the distribution.

\begin{tabular}{|c||c|c||c|c|}
\hline$\theta$ & $\tau_{c}, \exp$ & $\tau_{c}$, theory & $\xi_{c}, \exp$ & $\xi_{c}$, theory \\
\hline $19.87^{\circ}$ & $36 \pm 2 \mathrm{fs}$ & $28 \mathrm{fs}$ & $67 \pm 8 \mu \mathrm{m}$ & $59 \mu \mathrm{m}$ \\
\hline $19.90^{\circ}$ & $19 \pm 2 \mathrm{fs}$ & $22 \mathrm{fs}$ & $48 \pm 12 \mu \mathrm{m}$ & $46 \mu \mathrm{m}$ \\
\hline $19.94^{\circ}$ & $16 \pm 2 \mathrm{fs}$ & $17 \mathrm{fs}$ & $38 \pm 7 \mu \mathrm{m}$ & $37 \mu \mathrm{m}$ \\
\hline
\end{tabular}

Table 1: Coherence times and radiuses of PDC measured and calculated for different crystal orientations $(\theta)$ of the BBO crystal

Another feature noticeable in Fig. 4 is that the rings surrounding the central peak get more and more pronounced as the orientation gets further from collinear degenerate and the ring-shaped spectra become larger. Indeed, the height of the first ring is about 0.22 for orientation $19.90^{\circ}$ and 0.30 for orientation $19.94^{\circ}$. This again demonstrates that the coupling between temporal and spatial coherence occurs due to the non-factorability of the frequency transverse wavevector spectrum.

In conclusion, we have demonstrated the measurement of coupled spatiotemporal coherence, with the simultaneous control over space and time vari- ables. We applied this technique to the radiation of high-gain parametric down-conversion in the negative GVD range, where the frequency-wavevector spectra are ring-shaped. The obtained 2D distributions of the first-order correlation function demonstrate strong coupling between temporal and spatial degrees of freedom. Due to the large frequency and angular bandwidth of the radiation, and especially due to the absence of collinear frequency-degenerate emission, it is possible to achieve very short time and space coherence scales (on the order of femtoseconds and micrometers, respectively). The same experimental technique will be suitable for measuring the second-order correlation function and therefore implementing two-photon space-time nonlinear spectroscopy with PDC radiation.

We thank Denis Kopylov for the help in experiment.

\section{Funding Information}

P. Cutipa acknowledges the funding provided by the CONICYT PFCHA/DOCTORADO BECAS CHILE/2019 - 72180453.

\section{References}

[1] Rodney Loudon. Non-classical effects in the statistical properties of light. Reports on Progress in Physics, 43(7):913, 1980.

[2] Joseph W Goodman. Statistical optics. John Wiley \& Sons, 2015.

[3] Alessia Allevi, Ottavia Jedrkiewicz, Enrico Brambilla, Alessandra Gatti, J Peřina Jr, O Haderka, and Maria Bondani. Coherence properties of high-gain twin beams. Physical Review A, 90(6):063812, 2014.

[4] Leonard Mandel and Emil Wolf. Optical coherence and quantum optics. Cambridge university press, 1995.

[5] Dmitry Strekalov, Andrey B Matsko, Anatoliy A Savchenkov, and Lute Maleki. Relation- 
ship between quantum two-photon correlation and classical spectrum of light. Physical Review A, 71(4):041803, 2005.

[6] Ottavia Jedrkiewicz, Matteo Clerici, Antonio Picozzi, Daniele Faccio, and Paolo Di Trapani. X-shaped space-time coherence in optical parametric generation. Physical Review A, 76(3):033823, 2007.

[7] Enrico Brambilla, Ottavia Jedrkiewicz, Luigi Alberto Lugiato, and Alessandra Gatti. Disclosing the spatiotemporal structure of parametric down-conversion entanglement through frequency up-conversion. Physical Review A, 85(6):063834, 2012.

[8] Antonio Picozzi and Marc Haelterman. Skewed coherence ong space-time trajectories in parametric generation processes. In Nonlinear Guided Waves and Their Applications, page NLMB7. Optical Society of America, 2002.

[9] Ottavia Jedrkiewicz, Antonio Picozzi, Matteo Clerici, Daniele Faccio, and Paolo Di Trapani. Emergence of $\mathrm{x}$-shaped spatiotemporal coherence in optical waves. Physical review letters, 97(24):243903, 2006.

[10] Dmitry Strekalov, Andrey B Matsko, Anatoly Savchenkov, and Lute Maleki. Quantumcorrelation metrology with biphotons: where is the limit? Journal of Modern Optics, 52(16):2233-2243, 2005.

[11] Kirill Yu Spasibko, Denis A Kopylov, Tatiana V Murzina, Gerd Leuchs, and Maria V Chekhova. Ring-shaped spectra of parametric downconversion and entangled photons that never meet. Optics letters, 41(12):2827-2830, 2016.

[12] Konstantin E Dorfman, Frank Schlawin, and Shaul Mukamel. Nonlinear optical signals and spectroscopy with quantum light. Reviews of Modern Physics, 88(4):045008, 2016.
[13] O Jedrkiewicz, A Gatti, E Brambilla, and P Di Trapani. Experimental observation of a skewed x-type spatiotemporal correlation of ultrabroadband twin beams. Physical review letters, 109(24):243901, 2012.

[14] Fabien Boitier, Antoine Godard, Nicolas Dubreuil, Philippe Delaye, Claude Fabre, and Emmanuel Rosencher. Two-photon-counting interferometry. Physical Review A, 87(1):013844, 2013.

[15] Roberto de J León-Montiel, Jiří Svozilík, Juan P Torres, and Alfred B URen. Temperaturecontrolled entangled-photon absorption spectroscopy. Physical review letters, 123(2):023601, 2019.

[16] Jean-Philippe W MacLean, John M Donohue, and Kevin J Resch. Direct characterization of ultrafast energy-time entangled photon pairs. Physical review letters, 120(5):053601, 2018.

[17] Enrico Brambilla, Alessandra Gatti, Morten Bache, and Luigi A Lugiato. Simultaneous nearfield and far-field spatial quantum correlations in the high-gain regime of parametric downconversion. Physical Review A, 69(2):023802, 2004 .

[18] K Yu Spasibko, T Sh Iskhakov, and Maria V Chekhova. Spectral properties of high-gain parametric down-conversion. Optics express, 20(7):7507-7515, 2012.

[19] Angela M Pérez, Kirill Yu Spasibko, Polina R Sharapova, Olga V Tikhonova, Gerd Leuchs, and Maria V Chekhova. Giant narrowband twin-beam generation along the pump-energy propagation direction. Nature communications, $6(1): 1-5,2015$.

[20] David Nikolaevich Klyshko, Maria Chekhova, and Sergey Kulik. Physical foundations of quantum electronics. World Scientific, 2011. 